\title{
Analysis of system response and postgraduate entrance examination questions
}

\author{
LIAN Li-hong
}

Xiamen UniversityTanKahKeeCollege,Zhangzhou Campus of Xiamen University, 363105, China Ihlian@xujc.com

Keywords: Response solution; Classical method; Convolution method;Transform domain method.

Abstract. "Signal and system" is the key examination subject of entrance examination for Postgraduates of electronic communication specialty. System response calculation is the main line of "signal and system", so it is very important to study and master the relevant algorithm. Firstly, three algorithms of system response, classical method, convolution method and transformation domain method, are introduced. Next, combining with the entrance examination of Xiamen University, the main types of examination questions and solutions are discussed to provide some reference for the students. Finally, the applicability of the three methods is compared and analyzed.

\section{系统响应求解相关方法及考研题型分析}

\author{
连丽红 \\ 厦门大学嘉庚学院, 漳州, 福建, 中国 \\ Ihlian@xujc.com
}

关键词: 响应求解; 经典法; 卷积法; 变换域法

中文摘要. “信号与系统” 是电子通信专业考研的重点考核科目。系统响应求解则是 “信号 与系统” 的主线, 因此对其相关求解算法的研究及掌握至关重要。本文首先阐述了系统响应 求解三种算法一一时域经典法、卷积法和变换域法的求解思路, 接着结合厦门大学研究生入 门考试探讨主要的考核题型及求解方法, 为考研同学提供一定的参考, 最后对比分析三种方 法的适用场景。

\section{1. 引言}

方程求解作为理工科学生必须具备的基本技能之一，其应用广度已经几乎渗透到每一门 课程。在不同课程甚至同一门课程里, 方程都会有着不同的形式和附加条件, 其求解思路和 方式也会有所区别 ${ }^{[1-2]}$ 。因此, 学生在方程求解之前必须分析方程形式、熟悉初始条件才能确 保准备无误求解。“信号与系统” 围绕连续与离散两大系统展开, 微分、差分方程作为各自 的数学模型, 贯穿课程始终。两大方程的求解是课程的重点内容之一, 因此对其研究对课程 学习有着较强的指导意义。本文以连续系统的系统响应求解为例, 详细介绍其求解算法和研 究生入门考试的考核重点。 


\section{2. 系统响应求解}

\section{1 时域经典法}

时域经典法使用高等数学微分方程的求解思路, 其主要步骤如下:

(1) 完全解分成齐次解和特解;

(2) 列出特征方程, 求特征根, 写出齐次解形式;

（3）代入激励, 根据自由项求解特解;

（4）由初始条件求解待定系数, 得到完全解。

时域经典法注重物理意义, 因此信号与系统在微分方程求解基础上建立自由响应和强迫 响应、零输入响应和零状态响应、瞬态响应和稳态响应概等念。其中, 零输入响应和零状态 响应是根据系统响应产生的两个原因（系统的原始储能和输入激励信号）将系统完全响应分 解而来。系统零输入响应是在没有激励信号作用下产生的, 因此该反应不会随激励信号不同 而不同, 具有一定的不变性。系统的零状态响应相比于完全响应求解区别在系统起始状态为 0 , 因此使用经典法求零状态响应的复杂度等同于求完全响应。

此外, 经典法求解响应有别于高等数学微分方程求解最重要的一点是：由于冲激信号的 引入, 系统的初始状态 $r\left(0_{+}\right)$相对起始状态 $r\left(0_{-}\right)$可能会发生跳变。即: 微分方程在代入激励 信号后方程右边即 “自由项” 如果含有冲激信号, 则初始状态相对起始状态就会发生跳变, 此时可使用冲激函数匹配法进行计算。冲激函数匹配法的基本思路是在 $\mathrm{t}=0$ 时刻微分方程左右 两端的 $\delta(\mathrm{t})$ 及其各阶导数应该平衡相等。匹配从方程左端 $\mathrm{r}^{(\mathrm{k})}(\mathrm{t})$ 的最高阶项开始, 首先使方程右 端 $\delta$ 函数最高阶次项得到匹配, 然后匹配低阶 $\delta$ 函数项, 如果方程左端所有同阶次 $\delta$ 函数各项系 数之和不能和右端匹配, 则由左端 $\mathrm{r}^{(\mathrm{k})}(\mathrm{t})$ 最高阶项中补偿, 从而判断跳变值。

例：微分方程代入激励信号化简后如式（1）所示，可见 “自由项” 含有冲激信号，需要 进行匹配。根据匹配原理首先可以直接对 $\mathrm{r}(\mathrm{t})$ 及各阶导数带参数设值如式（2）所示; 接着代 入方程求解各参数 $(\mathrm{a}=3 ; \mathrm{b}=-9 ; \mathrm{c}=9)$, 最后由式 (2) 便可知 $r\left(0_{+}\right)$比 $r\left(0_{-}\right)$增加了 $\mathrm{b}$ 。

$$
\begin{gathered}
\frac{d r(t)}{d t}+3 r(t)=3 \delta^{\prime}(t) \\
\left\{\begin{array}{c}
\frac{d r(t)}{d t}=a \delta^{\prime}(t)+b \delta(t)+c \Delta u(t) \\
r(t)=a \delta(t)+b \Delta u(t)
\end{array}\right.
\end{gathered}
$$

\section{2 卷积法}

卷积积分是时域与频域的纽带, 其具体计算方法相关研究可以参考文献 ${ }^{[3-4]}$ 。此外, 如文 献 ${ }^{[5-6]}$ 基于卷积的神经网络算法在各领域也得到广泛应用。在时域领域求解上, 卷积积分需在 求得冲激响应 $\mathrm{h}(\mathrm{t})$ 的基础上, 利用式 (3) 求系统的零状态响应, 而系统零输入响应仍要用经 典法求得。

$$
r_{z s}(t)=\int_{-\infty}^{\infty} e(\tau) h(t-\tau) d \tau=e(t) * h(t)
$$

\section{3 变换域法}

拉普拉斯变换是时域与复频域相互转换的积分过程, 它能化微积分运算为乘除法运算, 大大降低计算难度, 得到广泛应用如文献 ${ }^{[7-8]}$ 。变换域法主要是利用拉普拉斯变换及逆变换将 微分方程先变换后求得变换解再逆变换求得时域解。例: 首先利用拉普拉斯变换的微分性质 对式 (4) 的微分方法方程左右两边进行变换, 接着化简求得响应的频域表达式式 (5), 再 代入系统的起始条件和激励信号的拉普拉斯变换 $\mathrm{F}(\mathrm{s})$ 化简 $\mathrm{Y}(\mathrm{s})$, 最后经过拉普拉斯逆变换求得 响应y(t)。 


\section{3. 考研题型及适用场景分析}

$$
\begin{gathered}
a_{n} y^{(n)}(t)+a_{n-1} y^{(n-1)}(t)+\cdots+a_{0}=b_{m} f^{(m)}(t)+\cdots+b_{0} \\
Y(s)=\frac{\sum_{i=0}^{n} a_{i}\left[\sum_{p=0}^{i-1} s^{i-1-p} y^{(p)}\left(0_{-}\right)\right]}{\sum_{i=0}^{n} a_{i} s^{i}}+\frac{\sum_{j=0}^{m} b_{j} s^{j}}{\sum_{i=0}^{n} a_{i} s^{i}} F(s)
\end{gathered}
$$

\section{1 直接考核题型}

直接考核题型是指题目中直接给出微分方程和起始条件, 要求求解各种响应。此类题型 相对基础, 近几年的考核概率较低, 主要集中在求冲激响应和阶跃响应。其中, 阶跃响应可 以直接由冲激响应的积分得到。冲激响应求解方法有两种: 一是用经典法求冲激响应; 一是 利用拉普拉斯变换求系统函数, 再逆变换求冲激响应。此外, 微分方程也出现过结合了卷积 积分的变型, 考生只要能结合式 (3) 进行简要化简, 便可转换将其转换为普通微分方程进行 求解。

\section{2 间接考核题型}

近几年来，系统响应的间接考核题型主要分为以下几种:

(1) 题目给出系统在两种激励下的完全响应, 要求求解系统在其它激励信号下的响应。 此类题型近几年的考核概率很高, 其最大特点是系统方程未知, 所以经典法和变换域法都没 法对其进行求解, 大部分只能使用卷积法进行求解。具体思路如下:

1) 如式 (6) 所示, 考生可以利用零输入响应在不同激励下不变的特点和卷积积分求零 状态响应的公式, 将系统响应分解成零输入响应和零状态响应。

$$
\begin{aligned}
& r_{1}(t)=r_{z i}(t)+r_{1 z s}(t)=r_{z i}(t)+e_{1}(t) * h(t) \\
& r_{2}(t)=r_{z i}(t)+r_{2 z s}(t)=r_{z i}(t)+e_{2}(t) * h(t)
\end{aligned}
$$

2)将激励信号和响应代入上式化简, 求得 $r_{z i}(t) 、 h(t)$ 的值;

$3)$ 利用式 $(7)$ 求 $r_{3}(t)$ 。

$$
r_{3}(t)=r_{z i}(t)+r_{3 z s}(t)=r_{z i}(t)+e_{3}(t) * h(t)
$$

(2) 题目给出电路图, 要求画出 $S$ 域电路图、并求解响应。此类型题目考核概率也较大, 主要使用变换域算法, 其一般有两种类型:

1) 题目中电容、电感器件的起始状态兼为 0 , 此类考题侧重考核系统函数, 此时等效 $S$ 域电路中电容、电感只需画出其等效阻值, 附加的电压源、电流源等都为 0 , 再根据基本电路 理论和系统函数定义进行相关计算，相对简单。

2 ) 题目中电容、电感器件的起始状态不为 0 , 此类考题还是以考核响应为主, 此时等效 $S$ 域电路中电容、电感除了画出等效阻值, 必须记得加上附加电压源、电流源等, 再根据基本 电路理论求解响应。

(3) 题目给出模拟框图, 要求求解响应。同 (2) 类似此类题型也侧重变换域算法, 其求 解基本都是跟系统函数有关。一般题目中的框图可能时域、频域交叠出现, 考生首先需要根 据卷积定理如式 (8) 将其统一化为频域框图, 接着列出 $S$ 域方程, 最后再根据需要进行相关 求解 (包括系统函数、稳定系统参数计算等)。

$$
f_{1}(t) * f_{2}(t) \stackrel{\mathrm{L}}{\longrightarrow} F_{1}(s) \bullet F_{2}(s)
$$

\section{3 适用场景分析}

由上可见, 系统响应的相关算法在其使用上有着各自的适应情景, 必须根据实际需求进 行选择, 具体如下: 
(1) 时域经典法主要适用于对给定微分方程直接求解响应的题型, 具有较强的物理意义, 便于理解, 但计算量一般相对较大;

（2）卷积法主要适应用于未给定微分方程求响应、已知冲激响应下求零状态响应、模拟 框图中时域变换域间相互转换等题型，使用较为灵活，利用好它，大多可以降低计算量;

(3) 变换域法既适用于对给定微分方程直接求解响应的题型, 又适用于各种电路、框图 求解响应的题型。

\section{4. 总结}

系统响应求解是系统性能判断的主要根据。针对不同的系统方程及给定条件, 寻求合适 的求解方法, 能有效地降低求解难度, 并提高求解准确度。本文在详细介绍 “信号与系统” 连续时间LTI系统三种求解算法的基础上, 重点分析了近几年的相关考核重点及解题思路, 对 考生有着较强地指导意义。

\section{References}

[1] ZHUANG Qingqu,WANG Jinping, Birkhoff Collocation Method for Fourth-Order Ordinary Differential Equations, Journal of Huaqiao University, vol. 39, pp. 306-310, 2018.

[2] LUO Shuang-hua,WANG Song,ZHANG Cheng-yi,LIU Qing-bing, Local Polynomial Smoother for Solving Bagley-Torvik Fractional Differential Equations,CHINESE JOURNAL OF ENGINEERING MATHEMATICS, vol. 35, pp. 88-99, 2018.

[3] WANG Zhongpeng, Exploration of case-based curriculum teaching of Fundamentals of Signal and System -in the case of convolution integral, Journal of Zhejiang Univiersity of Science and Technology, vol. 27, pp. 476-481, 2017.

[4] CHEN Ying-pin,CHENG Zhu-yuan,WANG Ling-zhi,YU Fei, LIU Xing-guo, The Computation of Convolution Based on the Dynamic Coordinate and Graphical Method, Journal of Minman Normal Univiersity, vol. 1, pp. 30-38, 2016.

[5] Long J,Shelhamer E,Darrell T.Fully convolutional networks for semantic segmentation[C] ,Proc of IEEE Conference on Computer Vision and Pattern Recognition. [S. 1. ]: IEEE Press,pp: 3431-3440,2015.

[6] LI Chuanpeng,QIN Pinle,ZHANG Jinji, Research on Image Denoising Based on Deep Covolutional Neural Network, Computer Engineering, vol43, pp. 253-260, 2017.

[7] QIN Xu-jia,KE Ling-ling,FAN Ying-lin,ZHENG Hong-bo,ZHANG Mei-yu, Improved Hyper-laplacian Constraint Single Image Deblurring Algorithm, Journal of Chinese Computer Systems, vol39, pp. 1097-1102, 2017.

[8] HUANG Taotao,GU Jingjing,ZHUANG Yi,Mobile Localization Algorithm Based on Semi-supervision Laplacian Mapping, Computer Engineering, vol44, pp. 144-153, 2018. 